\title{
Bounds for Completely Decomposable Jacobians
}

\author{
Iwan Duursma $^{1}$ and Jean-Yves Enjalbert ${ }^{2}$ \\ 1 University of Illinois at U-C, Urbana IL 61801, USA \\ 2 Université de Limoges, F-87060 Limoges Cedex, France
}

\begin{abstract}
A curve over the field of two elements with completely decomposable Jacobian is shown to have at most six rational points and genus at most 26 . The bounds are sharp. The previous upper bound for the genus was 145 . We also show that a curve over the field of $q$ elements with more than $q^{m / 2}+1$ rational points has at least one Frobenius angle in the open interval $(\pi / m, 3 \pi / m)$. The proofs make use of the explicit formula method.
\end{abstract}

\section{Introduction}

The Jacobian of an (absolutely irreducible, projective, non-singular) algebraic curve is said to be completely decomposable if it is isogenous over the base field to a product of elliptic curves. Many examples are known of curves with completely decomposable Jacobian ES93, both in characteristic zero and in finite characteristic. For a curve over a finite field $F_{q}$, the genus of a curve with completely decomposable Jacobian is bounded [TV97, Ser97. For $q=2$, Serre Ser97 gives a first order estimate $g<146$. We use the explicit formula method developed in Ser83 to obtain $g \leq 26$. The upper bound is sharp and is attained by the modular curve $X(11)$ for which Hecke showed that the Jacobian decomposes as $E_{1}^{5} \times E_{2}^{10} \times E_{3}^{11}$ [Lig77].

For an algebraic curve (absolutely irreducible, projective, non-singular) of genus $g$ over a finite field of $q$ elements, the Hasse-Weil bound gives that the number of rational points $N$ does not exceed $q+1+2 g \sqrt{q}$. For the explicit formula method, the number of rational points is expressed in terms of the Frobenius eigenvalues as

$$
N=q+1-\sum_{j=1}^{g}\left(\alpha_{j}+\bar{\alpha}_{j}\right) .
$$

By Weil's theorem, we may write $\alpha_{j}=\sqrt{q} e^{i \theta_{j}}$, for elements $\theta_{j}$ in $[0, \pi]$ for all $j$. The $\theta_{j}$ are called the Frobenius angles. Over an extension field of size $q^{m}$, the number of rational points $N_{m}$ is given by

$$
N_{m}=q^{m}+1-\sum_{j=1}^{g}\left(\alpha_{j}^{m}+\bar{\alpha}_{j}^{m}\right)=q^{m}+1-r^{m} \sum_{j=1}^{g} 2 \cos m \theta_{j},
$$


where $r=\sqrt{q}$. For curves of large genus, the distribution of the Frobenius angles is restricted by the constraints $N_{d m} \geq N_{m}$, for all $m, d$. This allows one to obtain upper bounds of the form $N \leq a g+b$ for the number of rational points that are better than the Hasse-Weil bound when the genus is large Iha81, Ser83. Asymptotically, the Drinfeld-Vladuts bound gives $\lim \sup _{g \rightarrow \infty} N / g \leq \sqrt{q}-1$ [VD83], where the limit is over an infinite family of curves of increasing genus. In Section 2.1, we recall the main steps of the explicit formula method.

Tsfasman-Vladuts [TV97] and Serre [Ser97] study the distribution of the Frobenius angles for families of curves of increasing genus. It is easy to see that any infinite family of curves of increasing genus contains a subfamily for which $N_{m} / g$ approaches a limit, for each $m$, when the genus increases. Such subfamilies are called asymptotically exact in TV97. For curves in an asymptotically exact family, the distribution of the Frobenius angles approaches a limit distribution that is given by a continuous measure on $[0, \pi]$. In particular, the Frobenius angles in an asymptotically exact family are dense in $[0, \pi]$. This shows that any family of curves for which the Frobenius angles are not dense in $[0, \pi]$ is finite. We consider the following problem.

(Problem 1) Given a discrete subset $\Theta$ of $[0, \pi]$, maximize $N$ and $g$ for a curve over $F_{q}$ with all Frobenius angles in $\Theta$.

The elliptic curves over the field of two elements have Frobenius angle $\theta$ such that $2 \sqrt{2} \cos \theta \in\{-2,-1,0,1,2\}$. The corresponding Frobenius eigenvalues are of degree at most two. As a special case of the previous problem we have

(Problem 2) Maximize $N$ and $g$ for a curve over $F_{q}$ with all Frobenius eigenvalues of bounded degree at most $d$.

The case $d=2$ corresponds to curves with completely decomposable Jacobian. In Section 2.3 and Section 2.4 respectively, we show that a curve over $F_{2}$ with completely decomposable Jacobian has $N \leq 6$ and $g \leq 26$, respectively. Similarly, the family of curves with no Frobenius angle in a given interval is finite. And we can ask for the largest number of rational points or the largest genus for curves in the family.

(Problem 3) Given a (small) subset $I$ of $[0, \pi]$, maximize $N$ and $g$ for a curve over $F_{q}$ with all Frobenius angles outside $I$.

In Section 3, we prove that any curve over $F_{q}$ with $N>q^{m / 2}+1$ has a Frobenius angle in the open interval $(\pi / m, 3 \pi / m)$. We formulate one other problem along the same lines. It will not be considered in this paper however.

(Problem 4) Given $\delta$, maximize $N$ and $g$ for a curve over $F_{q}$ such that $[0, \pi] \not \subset \cup_{j}\left(\theta_{j}-\delta, \theta_{j}+\delta\right)$. 


\section{The explicit formula method}

We first recall the explicit formula method and its use in obtaining general upper bounds for the number of rational points on a curve Ser83, Han95. Then we present three variations of the method that yield better bounds for curves whose Frobenius angles are restricted to a subset $\Theta$ of $[0, \pi]$. In particular, curves that exceed one of the latter bounds, necessarily have at least one Frobenius angle outside $\Theta$.

\subsection{General upper bounds for the number of rational points}

For an algebraic curve $X$ of genus $g$ over the finite field $F_{q}$ of $q$ elements, let the Frobenius angles be $\theta_{1}, \theta_{2}, \ldots, \theta_{g}$. So that the number of rational points $N_{n}$ over $F_{q^{n}}$ satisfies

$$
N_{n}=q^{n}+1-q^{n / 2} \sum_{j=1}^{g} 2 \cos n \theta_{j}
$$

With $r=\sqrt{q}$, we rewrite the equation as

$$
N_{1} r^{-n}+\left(N_{n}-N_{1}\right) r^{-n}=r^{n}+r^{-n}-\sum_{j=1}^{g} 2 \cos n \theta_{j} .
$$

Let $f$ be an auxiliary cosine polynomial with real coefficients $u_{n}$,

$$
f(\theta)=u_{0}+\sum_{n \geq 1} u_{n} \cos n \theta .
$$

Define

$$
\psi(x)=\sum_{n \geq 1} u_{n} x^{n} .
$$

The equations (10) scaled by $u_{n}$, for $n=1,2, \ldots$, add up to

$$
\begin{aligned}
N_{1} \psi\left(r^{-1}\right)+\sum_{n \geq 2} u_{n}\left(N_{n}-N_{1}\right) r^{-n} & = \\
& =2 u_{0} g+\psi(r)+\psi\left(r^{-1}\right)-2 \sum_{j=1}^{g} f\left(\theta_{j}\right) .
\end{aligned}
$$

The equation (4) leads to upper bounds for the number of points. As in Ser83, choose $\left\{u_{n}\right\}$ such that $u_{0}=1$, and

(a) $u_{n} \geq 0, \forall n \geq 1$

(b) $f(\theta) \geq 0$, for all $\theta \in[0, \pi]$. 
Then Equation (4) yields

$$
N \psi\left(r^{-1}\right) \leq 2 g+\psi\left(r^{-1}\right)+\psi(r) .
$$

As an example, the choice

$$
\begin{aligned}
f(\theta) & =\cos ^{2} \theta\left(1-\cos \theta / \cos \left(\frac{5 \pi}{6}\right)\right)^{2} \\
& =1+\sqrt{3} \cos \theta+\frac{7}{6} \cos 2 \theta+\frac{\sqrt{3}}{3} \cos 3 \theta+\frac{1}{6} \cos 4 \theta
\end{aligned}
$$

gives, for $q=3$, the upper bound

$$
N \leq \frac{54}{41}(g-15)+28<1.317 g+8.244 .
$$

This is better than the Hasse-Weil bound $N \leq 2 \sqrt{3} g+4$ for all $g \geq 2$. A curve attains the upper bound above only if $N_{1}=N_{2}=N_{3}=N_{4}$ and if all its Frobenius angles are among $\{\pi / 2,5 \pi / 6\}$. The unique such curve is the Deligne-Lusztig curve associated to ${ }^{2} G_{2}(3)$ HP93. The curve is of genus $g=15$ and has $N=28$. Its zeta function $Z(T)=P(T) /(1-T)(1-3 T)$ has numerator $P(T)=\left(1+3 T^{2}\right)^{7}\left(1+3 T+3 T^{2}\right)^{8}$.

\subsection{Restricted upper bounds for the number of rational points $\left(u_{0}=1\right)$}

The upper bound in the previous subsection generalizes as follows. Choose $\left\{u_{n}\right\}$ in Equation (4) such that

(a) $u_{0}=1$ and $u_{n} \geq 0, \forall n \geq 2$.

(b) $f(\theta) \geq 0$, for all $\theta \in \Theta \subset[0, \pi]$.

Then, for a curve that has all its Frobenius angles contained in $\Theta$,

$$
N \psi\left(r^{-1}\right) \leq 2 g+\psi\left(r^{-1}\right)+\psi(r) .
$$

The converse yields that a curve with

$$
N \psi\left(r^{-1}\right)>2 g+\psi\left(r^{-1}\right)+\psi(r) .
$$

has a Frobenius angle outside $\Theta$. For $0<\alpha<\beta<\pi$, let

$$
\begin{aligned}
f_{2}(\theta) & =(\cos \theta-\cos \alpha)(\cos \theta-\cos \beta), \\
& =\frac{1}{2}+\cos \alpha \cos \beta-(\cos \alpha+\cos \beta) \cos \theta+\frac{1}{2} \cos 2 \theta .
\end{aligned}
$$

Then $f_{2}(\theta)$ is non-negative on $\Theta=[0, \pi] \backslash(\alpha, \beta)$. For $q=2$, and for $\alpha=\pi / 3$ and $\beta=3 \pi / 4$, we obtain

$$
N>\frac{8-2 \sqrt{2}}{7}(g-1)+5 \Rightarrow \exists \theta_{j} \in\left(\frac{\pi}{3}, \frac{3 \pi}{4}\right) .
$$


The inequality on the left applies in the range $2 \leq g \leq 38$. In that range the inequality holds for a curve that meets the Oesterlé upper bound for the number of points. For another example, let

$$
\begin{aligned}
f(\theta) & =(1+\sqrt{2} \cos \theta)(1-2 \sqrt{2} \cos \theta)^{2}, \\
& =1+3 \sqrt{2} \cos \theta+2 \sqrt{2} \cos 3 \theta .
\end{aligned}
$$

We obtain, for a curve over $F_{2}$,

$$
N>\frac{1}{2}(g-1)+5 \quad \Rightarrow \quad \exists \theta_{j} \in\left(\frac{3 \pi}{4}, \pi\right] .
$$

\subsection{Uniform upper bounds for the number of rational points $\left(u_{0}=0\right)$}

By choosing $u_{0}=0$, we obtain upper bounds for the number of rational points that are independent of the genus $g$. Choose $\left\{u_{n}\right\}$ in Equation (4) such that

(a) $u_{0}=0$ and $u_{n} \geq 0, \forall n \geq 2$.

(b) $f(\theta) \geq 0$, for all $\theta \in \Theta \subset[0, \pi]$.

Then the number $N$ of rational points on a curve with all Frobenius angles contained in $\Theta$ satisfies

$$
N \psi\left(r^{-1}\right) \leq \psi\left(r^{-1}\right)+\psi(r) .
$$

If, moreover, the coefficients $u_{n}$ have the following symmetry property, for some positive integer $m$ with $m>\operatorname{deg}(\psi)$,

(c) $u_{n}=u_{m-n}$, for $n=0,1, \ldots, m$,

then the upper bound becomes

$$
N \leq 1+\frac{\sum_{n=0}^{m} u_{n} r^{n}}{\sum_{n=0}^{m} u_{m-n} r^{n-m}}=r^{m}+1 .
$$

The function

$$
\begin{aligned}
f(\theta) & =\frac{\sqrt{2}}{5} \cos \theta\left(1-2 \cos ^{2} \theta\right)\left(1-8 \cos ^{2} \theta\right) \\
& =\frac{7}{10} \sqrt{2} \cos \theta+\frac{1}{2} \sqrt{2} \cos 3 \theta+\frac{1}{5} \sqrt{2} \cos 5 \theta
\end{aligned}
$$

cancels at the Frobenius angles of the five different elliptic curves over $F_{2}$. It leads to the bound $N \leq 6$ for any curve $X$ over $F_{2}$ with completely decomposable Jacobian. The bound is tight only when $N_{1}=N_{3}=N_{5}$. The 
smallest feasible zeta function is of genus 3 with uniquely determined zeta polynomial $P(T)=\left(1+2 T+2 T^{2}\right)^{2}\left(1-T+2 T^{2}\right)$. It is realized by the curve

$$
y^{2}+y=\frac{x^{2}+x}{\left(x^{2}+x+1\right)^{3}} \text {. }
$$

We give two examples that use Condition (c). The choice $f(\theta)=\cos \theta$ yields that a curve with $N>r^{2}+1$ has a Frobenius angle in $(\pi / 2,3 \pi / 2)$ (indeed the Frobenius trace can only be negative if at least one Frobenius angle has $\cos \theta<0)$. The choice $f(\theta)=\cos \theta+\cos 2 \theta$ yields that a curve with $N>r^{3}+1$ has a Frobenius angle in $(\pi / 3, \pi)$. In both cases, the bound on $N$ is sharp. The projective line with $N=r^{2}+1$ has no Frobenius angle in $(\pi / 2,3 \pi / 2)$, and the Hermitian curve (see [RS94]) over $F_{r^{2}}$ with $N=r^{3}+1$ has no Frobenius angle in $(\pi / 3, \pi)$. The latter example confirms that the Hasse-Weil bound is not sharp for curves with $N>r^{3}+1$. In Section 3] we show more generally that a curve with $N>r^{m}+1$ has a Frobenius angle in $(\pi / m, 3 \pi / m)$.

\subsection{Uniform upper bounds for the genus $\left(u_{0}=-1\right)$}

By choosing $u_{0}=-1$, we obtain upper bounds for the genus $g$. Choose $\left\{u_{n}\right\}$ in Equation (4) such that

(a) $u_{0}=-1$ and $u_{n} \geq 0, \forall n \geq 2$.

(b) $f(\theta) \geq 0$, for all $\theta \in \Theta \subset[0, \pi]$.

Then the genus of a curve with all Frobenius angles contained in $\Theta$ satisfies

$$
N \psi\left(r^{-1}\right)+2 g \leq \psi(r)+\psi\left(r^{-1}\right) .
$$

If, moreover, the coefficients $u_{n}$ satisfy

(d) $\psi\left(r^{-1}\right)=0$,

then the upper bound becomes

$$
2 g \leq \psi(r)
$$

The function

$$
f(\theta)=-1-\frac{4}{3} \cos \theta+\frac{7}{9} \cos 2 \theta+\frac{26}{9} \cos 3 \theta+\frac{16}{9} \cos 4 \theta
$$

is of minimal degree such that it cancels at the Frobenius angles of the three elliptic curves over $F_{4}$ that are defined over $F_{2}$ and such that Condition (d) holds. It leads to the bound $2 g \leq 52$ for any curve $X$ over $F_{2}$ with completely decomposable Jacobian. A previous estimate showed that $g \leq 145$ [Ser97. The bound is tight only when $N_{1}=N_{2}=N_{3}=N_{4}$ for the base field $F_{4}$. It is attained by the modular curve $X(11)$, which has $g=26, N=55$ over $F_{4}$, and zeta polynomial $P(T)=\left(1+4 T+4 T^{2}\right)^{5}\left(1+3 T+4 T^{2}\right)^{10}\left(1+4 T^{2}\right)^{11}$. 


\section{An asymptotic example}

Let $m \geq 4$ and let $\alpha=\pi / m$. Conditions (a)-(c) in Section 2.3 hold with $\Theta=[0, \pi] \backslash(\pi / m, 3 \pi / m)$ for coefficients $\left\{u_{n}\right\}$ that are defined by

$$
f(\theta)=\frac{1+\cos m \theta}{4(\cos \theta-\cos \alpha)(\cos \theta-\cos 3 \alpha)}=\sum_{n=2}^{m-2} u_{n} \cos n \theta .
$$

So that

$$
u_{n}=\frac{\sin (n-1) \alpha \sin n \alpha \sin (n+1) \alpha}{\sin \alpha \sin 2 \alpha \sin 3 \alpha}, \quad n=0,1, \ldots, m .
$$

Thus, a curve with number of rational points $N>r^{m}+1$, for $m \geq 4$, has at least one Frobenius angle in the open interval $(\pi / m, 3 \pi / m)$. For $f(\theta)$ we may write

$$
f(\theta)=2^{m-3} \prod_{k=2}^{m-1}(\cos \theta-\cos (2 k+1) \alpha),
$$

which justifies writing the right hand side of (5) as a cosine polynomial. To see that the coefficients of the cosine polynomial are those given by (6), we use a generating function for gaussian polynomials And98.

$$
\frac{1}{(1-T)(1-y T)\left(1-y^{2} T\right)\left(1-y^{3} T\right)}=\sum_{i \geq 0}\left[\begin{array}{c}
i+3 \\
3
\end{array}\right] T^{i}
$$

where

$$
\left[\begin{array}{c}
i+3 \\
3
\end{array}\right]=\frac{\left(y^{i+3}-1\right)\left(y^{i+2}-1\right)\left(y^{i+1}-1\right)}{\left(y^{3}-1\right)\left(y^{2}-1\right)(y-1)} .
$$

For $y$ with $y^{m}=1$, the right hand side is periodic and, for $n=i+2$,

$$
\frac{T^{2}\left(1-T^{m}\right)}{(1-T)(1-y T)\left(1-y^{2} T\right)\left(1-y^{3} T\right)}=\sum_{n=2}^{m-2} \frac{\left(y^{n+1}-1\right)\left(y^{n}-1\right)\left(y^{n-1}-1\right)}{\left(y^{3}-1\right)\left(y^{2}-1\right)(y-1)} T^{n} .
$$

Let $x=e^{i \alpha}$, so that $x^{m}=-1$. With $y=x^{2}$ and $t=x^{3} T$, we obtain

$$
\frac{\left(1+t^{m}\right)}{\left(t+t^{-1}-2 \cos \alpha\right)\left(t+t^{-1}-2 \cos 3 \alpha\right)}=\sum_{n=2}^{m-2} \frac{\sin (n-1) \alpha \sin n \alpha \sin (n+1) \alpha}{\sin \alpha \sin 2 \alpha \sin 3 \alpha} t^{n} .
$$

Now sum the two equations with $t=e^{i \theta}$ and $t=e^{-i \theta}$, respectively, and divide by 2 .

The cases $m=2$ and $m=3$ were considered in Section 2.3, so that the claim extends to all $m \geq 2$. For $m=4$ and $m=6$ the bounds are sharp, as can be seen by considering curves of Suzuky type or Ree type, respectively. The Suzuki curve over $F_{8}$ has $N=65$ but has no Frobenius angle in $(\pi / 4,3 \pi / 4)$. The Ree curve over $F_{3}$ has $N=28$ but has no Frobenius angle in $(\pi / 6, \pi / 2)$. 


\section{Conclusion}

Results by Tsfasman-Vladuts and Serre led us to consider Problems (1)(4) in the Introduction. For Problems (1)-(3) we have given methods that yield partial results. One result is a sharp upper bound for the number of points $(N \leq 6)$ or the genus $(g \leq 26)$ for a curve over $F_{2}$ with completely decomposable Jacobian. We also showed that a curve over $F_{q}$ with $N>$

$q^{m / 2}+1$ has at least one Frobenius angle in the interval $(\pi / m, 3 \pi / m)$. No results were obtained towards Problem (4).

\section{References}

And98. George E. Andrews. The theory of partitions. Cambridge University Press, Cambridge, 1998. Reprint of the 1976 original.

ES93. Torsten Ekedahl and Jean-Pierre Serre. Exemples de courbes algébriques à jacobienne complètement décomposable. C. R. Acad. Sci. Paris Sér. I Math., 317(5):509-513, 1993.

Han95. Søren Have Hansen. Rational points on curves over finite fields. Aarhus Universitet Matematisk Institut, Aarhus, 1995.

HP93. Johan P. Hansen and Jens Peter Pedersen. Automorphism groups of Ree type, Deligne-Lusztig curves and function fields. J. Reine Angew. Math., 440:99-109, 1993.

Iha81. Yasutaka Ihara. Some remarks on the number of rational points of algebraic curves over finite fields. J. Fac. Sci. Univ. Tokyo Sect. IA Math., 28(3):721724 (1982), 1981.

Lig77. Gérard Ligozat. Courbes modulaires de niveau 11. In Modular functions of one variable, V (Proc. Second Internat. Conf., Univ. Bonn, Bonn, 1976), pages 149-237. Lecture Notes in Math., Vol. 601. Springer, Berlin, 1977.

RS94. Hans-Georg Rück and Henning Stichtenoth. A characterization of Hermitian function fields over finite fields. J. Reine Angew. Math., 457:185-188, 1994.

Ser83. Jean-Pierre Serre. Sur le nombre des points rationnels d'une courbe algébrique sur un corps fini. C. R. Acad. Sci. Paris Sér. I Math., 296(9):397-402, 1983.

Ser97. Jean-Pierre Serre. Répartition asymptotique des valeurs propres de l'opérateur de Hecke T . J. Amer. Math. Soc., 10(1):75-102, 1997.

Tsf92. Michael A. Tsfasman. Some remarks on the asymptotic number of points. In Coding theory and algebraic geometry (Luminy, 1991), pages 178-192. Springer, Berlin, 1992.

TV97. M. A. Tsfasman and S. G. Vlăduţ. Asymptotic properties of zeta-functions. J. Math. Sci. (New York), 84(5):1445-1467, 1997. Algebraic geometry, 7.

VD83. S. G. Vlădut and V. G. Drinfeld̦. The number of points of an algebraic curve. Funktsional. Anal. i Prilozhen., 17(1):68-69, 1983. 


\section{Addendum : The modular curve $X(11)$ modulo 2}

The main text uses the following claim.

Claim: The modular curve $X(11)$ of genus 26 has a model over $\mathbb{F}_{2}$ with 55 rational points over $\mathbb{F}_{4}$.

The claim follows from results by Klein together with the following fact about the group $P S L(2,11)$.

Fact 1: The subgroups of order 12 in $P S L(2,11)$ divide into two conjugacy classes, each consisting of 55 copies of $A_{4}$. In each case the group $A_{4}$ is normally closed in $P S L(2,11)$.

The 55 rational points over $\mathbb{F}_{4}$ on $X(11)$ are the points above $j=0$ in the galois cover $X(11) \longrightarrow X(1)$ of degree 660 with group $P S L(2,11)$. Let the cover be defined over a finite field $k$ of characteristic 2 such that all automorphisms have their coefficients in $k$. Fact 1 shows that the stabilizer of a point above $j=0$ is a subgroup $A_{4}$ and moreover, since $A_{4}$ is normally closed, that the point is the unique fixed point of the stabilizer. In particular, the points above $j=0$ are defined over $k$.

With the previous argument it suffices to find a model for $X(11)$ defined over $\mathbb{F}_{2}$ such that the automorphisms are defined over $\mathbb{F}_{4}$. As starting point, we use Klein's model for $X(11)$ in $\mathbb{P}^{4}(\mathbb{C})$.

Fact 2 [Klein, Ges. Math. Abh. III, p.146]: Klein's model for $X(11)$ is defined over $\mathbb{Q}$ and has all its 660 automorphisms defined over $\mathbb{Q}(\rho)$, where $\rho$ is a primitive 11-th root of unity. The group contains the cyclic automorphism $C(v: w: x: y: z)=(z: v: w: x: y)$. Let $F$ be the automorphism of $\mathbb{Q}(\rho)$ with $F(\rho)=\rho^{4}$. The generators $S$ (of order 11) and $T$ (of order 2) presented by Klein satisfy $F(S)=C^{-1} \circ S \circ C$ and $F(T)=C^{-1} \circ T \circ C$.

A change of variables $(v: w: x: y: z)=Q\left(v^{\prime}: w^{\prime}: x^{\prime}: y^{\prime}: z^{\prime}\right)$ leads to a new model in the variables $\left(v^{\prime}: w^{\prime}: x^{\prime}: y^{\prime}: z^{\prime}\right)$. The new model is defined over $\mathbb{Q}$ if the set $\left\{Q, C \circ Q, C^{2} \circ Q, C^{3} \circ Q, C^{4} \circ Q\right\}$ is defined over $\mathbb{Q}$. This is achieved by defining $Q$ over $\mathbb{Q}\left(\rho+\rho^{-1}\right)$ such that $F(Q)=C^{i} \circ Q$, for some $i \in\{0,1,2,3,4\}$. After the change of variables, the automorphims are generated by $Q^{-1} \circ S \circ Q$ and $Q^{-1} \circ T \circ Q$. For a choice of $Q$ with $F(Q)=C^{-1} \circ Q$, the automorphisms are defined over the fixed field of $F$ : $F\left(Q^{-1} \circ A \circ Q\right)=Q^{-1} \circ A \circ Q$, for $A=S, T$.

Klein's model has good reduction modulo 2 and the twisted model is defined over $\mathbb{F}_{2}$ such that all automorphisms are defined over $\mathbb{F}_{4}$. 